Pacific Journal of Mathematics

ON SEMIPRIME P.I.-ALGEBRAS OVER COMMUTATIVE 


\title{
ON SEMIPRIME P.I.-ALGEBRAS OVER COMMUTATIVE REGULAR RINGS
}

\author{
Efraim P. ARMENDARIZ
}

Let $R$ be a commutative (von Neumann) regular ring with unit. This paper deals with algebras $A$ over $R$, and following standard conventions $A$ will be called a finitely generated $R$-algebra whenever $A$ is a finitely generated $R$-module. One of the principal results obtained is that all semiprime finitely generated $R$-algebras are regular rings. Combining this with a result of $J$. Wehlen and a theorem of G. Michler and 0 . Villamayor shows that the finitely generated semiprime algebras over commutative regular rings are precisely the semiprime central separable algebras over regular rings.

Since any finitely generated algebra over a commutative ring satisfies a polynomial identity, (is a P.I.-algebra), this leads to consideration of semiprime P.I.-algebras with regular center. In general, these will only be semisimple $I$-rings. However if the center is also a self-injective ring then the algebra is $\pi$-regular; this fact is a consequence of the observation that every semiprime P.I.-algebra is weakly algebraic over its center in the sense that every element is a root of a nonzero polynomial with central coefficients.

It will be assumed throughout that the polynomial identities occurring have at least one coefficient \pm 1 .

THEOREM 1. Let A be a semiprime finitely generated algebra over a commutative regular ring $R$. Then $A$ is a regular ring.

Proof. For the proof we appeal to a theorem of J. Fisher and R. Snider [5, Theorem 1.1] which says that $A$ will be a regular ring provided

(i) $A / P$ is a regular ring for each prime ideal $P$ of $A$,

(ii) the union of any chain of semiprime ideals of $A$ is a semiprime ideal of $A$.

For (i), we first observe that any homomorphic image of $R$ is a regular ring and hence we may assume that $A$ is a faithful $R$-algebra with $R$ lying in the center of $A$. Now if $P$ is any prime ideal of $A$ then $P \cap R$ is a prime ideal of $R$ hence is a maximal ideal of $R$. Consequently, $A / P$ is a prime finite-dimensional algebra over the field $R / P \cap R$ and so $A / P$ is a simple Artinian ring. Thus (i) is satisfied. For (ii) suppose $A=$ $R a_{1}+\cdots+R a_{t}$, let $\left\{B_{\lambda} \mid \lambda \in \Lambda\right\}$ be a chain of semiprime ideals of $A$ and let $B=\cup_{\lambda \in \Lambda} B_{\lambda}$. Let $x \in A$ with $x A x \subseteq B$. Then there is some 
$\lambda \in \Lambda$ such that $x a_{i} x \in B_{\lambda}$ for $i=1, \cdots, t$ and so $x A x \subseteq B_{\lambda}$. Hence $x \in B_{\lambda} \subseteq B$ since each $B_{\lambda}$ is a semiprime ideal. Thus $B$ is a semiprime ideal. It follows that $A$ is a regular ring.

Combining Theorem 1 with [12, Theorem 2.3] and [10, Theorem 6.3] we have the following description of finitely generated semiprime algebras over commutative regular rings.

THEOREM 2. Let $A$ be a finitely generated algebra over a regular ring. The following conditions on $A$ are equivalent:

(i) $A$ is semiprime.

(ii) $A$ is regular.

(iii) $A$ is biregular.

(iv) $A$ is a semiprime central separable algebra.

Proof. The equivalence of (ii) and (iii) is [10, Theorem 6.3] while the equivalence of (iii) and (iv) is [12, Theorem 2.3].

Wehlen has also shown [12, Corollary 2.3.1] that a finitely generated biregular $R$-algebra $A$ is separable over $R$ if and only if its center is separable over $R$. If $A$ is a finitely generated semiprime $R$-algebra with center $K$ then since $A$ is $K$-separable, $K$ is a $K$-direct summand of $A$ [4, Lemma 3.1, p. 51] hence $K$ is an $R$-direct summand of $A$. Thus $K$ is also a finitely generated $R$-module. On the other hand let $A$ be a semiprime $R$-algebra with $R$ regular whose center $K$ is a finitely generated $R$-module. Then $K$ is regular by Theorem 1 . If in addition $A$ is $K$-separable then $A$ is biregular and finitely generated over $K$ [12, Theorem 2.3]. We thus have

COROLlARY 1. Let $A$ be a semiprime $R$-algebra over a regular ring $R$. Then $A$ is a finitely generated and hence regular $R$-algebra if and only if $A$ is a central separable algebra whose center is a finitely generated $R$-module.

In terms of Hochschild dimension, $\operatorname{dim}_{R} A$, of $R$-algebras $A$ we have via [12, Corollary 2.4]

COROllary 2. Let $A$ be a finitely generated semiprime algebra over a regular ring $R$. Then $\operatorname{dim}_{R} A=0$ or $\operatorname{dim}_{R} A=\infty$.

It may be of some interest to know whether or not commutative regular rings $R$ are characterized by the property $\operatorname{dim}_{R} A=0$ or $\infty$ for all semiprime finitely generated $R$-algebras $A$.

Wehlen has also noted that a finitely generated algebra $A$ over a regular ring $R$ has nilpotent and finitely generated Jacobson radical $J(A)$ [12, Proposition 2.2]. 
COROLLARY 3. If $A$ is a finitely generated algebra over a regular ring $R$ then $J(A)$ is nilpotent and $A / J(A)$ is a regular ring.

In another direction we consider max-rings; a ring $K$ is a (left) max-ring if and only if each nonzero left $K$-module has a maximal submodule. Commutative max-rings have been characterized by $R$. Hamsher [6] as rings $K$ for which $J(K)$ is $T$-nilpotent and $K / J(K)$ is regular. We obtain a similar result for finitely generated algebras over max-rings, using Theorem 1.

COROLLARY 4. If $A$ is a finitely generated algebra over a max-ring, $R$ then $A$ is a left and right max-ring. Thus $J(A)$ is left and right $T$-nilpotent and $A / J(A)$ is a regular ring.

Proof. We let $M \neq 0$ be a left $A$-module and assume that $A$ is a faithful $R$-algebra with $R \subseteq$ center $A$. Since $R$ is a max-ring, $J(R) M \neq M[3]$, so we can assume that $J(R) M=0$. Then $M$ is a nonzero $R / J(R)$-module as well as an $A / J(R) A$-module. Since $A / J(R) A$ is an $R / J(R)$-algebra we can assume $J(R) A=0$ so $J(R)=$ 0 . By Corollary $3, J(A)$ is nilpotent and so $J(A) M \neq M$. By Theorem $1, A / J(A)$ is regular and hence by $[1$, Theorem 2(a)] $M / J(A) M$ has a maximal submodule. That $J(A)$ is left and right $T$-nilpotent results from [3, pp. 470-471].

Now we consider semiprime P.I. rings having regular center. There exist semiprime P.I. rings which are $\pi$-regular but not regular [5, Example 1]; such P.I. rings have regular center. An example in [2] shows that semiprime P.I. rings can have regular center without being $\pi$-regular. However that example is not a ring with identity, so we now provide one with an identity.

Let $F(t)$ be the rational functions over a field $F$, let

$$
K=\left(\begin{array}{ll}
F(t) & F(t) \\
F(t) & F(t)
\end{array}\right) \text {, and } L=\left(\begin{array}{cc}
F[t] & F(t) \\
0 & F
\end{array}\right) .
$$

Now if $A$ consists of all sequences with entries from $K$ which are eventually constant and in $L$, then $A$ is semiprime with identity and has regular center. Also $A$ is not $\pi$-regular since $A$ maps onto $F[t] \oplus F$ which is not $\pi$-regular.

We will show that if the center is also self-injective then $\pi$-regularity follows. This is a consequence of the following result which may be of some independent interest.

THEOREM 3. Let $A$ be a semiprime P.I. ring with center $R$. Then every element in A satisfies a nonzero polynomial with coefficients from 
R. Moreover if $a \in A$ is nilpotent then $p(x)=\Sigma_{i=0} r_{i} x^{i} \in R[X]$ can be chosen so that $r_{k} a^{k} \neq 0$ and $p(a)=0$.

Proof. Let $Q(A)$ be the maximal quotient ring of $A$ and $Q(R)$ the maximal quotient ring of $R$. By [2, Theorem 2.5 and Theorem 3.7] we have $Q(R)=$ center of $Q(A)$ and $Q(A)$ is a finitely generated $Q(R)$ module. Let $a \in A$; if $a$ is nilpotent then of course $a$ satisfies a monic polynomial in $R[X]$ so assume $a$ is not nilpotent. We consider $a$ as a $Q(R)$-homomorphism from $Q(A)$ into $Q(A)$ so by the Cayley-Hamilton theorem for finitely generated modules over a commutative ring, $a$ satisfies a monic polynomial in $Q(R)[x]$, say $a^{k}+$ $u_{k-1} a^{k-1}+\cdots+u_{1} a+u_{0}=0$. Since $Q(R)$ is the maximal quotient ring of $R$, there is an essential ideal $D$ of $R$ such that $D u_{i} \subseteq R$ for $0 \leqq i \leqq k-1$. Further $A D$ is essential in $A$ [19, Theorem 6] hence $A D a^{k} \neq 0$ so $D a^{k} \neq 0$. Hence choosing $r=r_{k} \in D$ so that $r a^{k} \neq 0$ and letting $r_{i}=r u_{i}, 1 \leqq i \leqq k-1$, we see that $a$ is a root of $p(x)=\sum_{i=0}^{k} r_{i} x^{i}$ with $r_{k} a^{k} \neq 0$.

Observe that the degree $k$ of the polynomial satisfied by $a$ is independent of $a$ since, as the proof shows, $k$ is at most the number of generators for $Q(A)$ over $Q(R)$. From the proof we deduce the following:

THEOREM 4. If $A$ is a semiprime P.I. ring whose center $R$ is $a$ self-injective ring then $A$ is integral over $R$, hence $A$ is a $\pi$-regular ring.

Proof. $Q(R)=R$ so $A$ is integral over $R$. It follows that primes of $A$ are maximal [7] so $A$ is $\pi$-regular [5, Theorem 2.3].

We now show how to obtain rings as in Theorem 4. Let $A$ be any semiprime P.I. ring with center $R$ and let $C=Q(R)$ so that $C$ is a regular self-injective ring. In $Q(A)$ we form the ring $S=$ $A C$. Following Martindale [8] we call $S$ the central closure of $A$. In general $S \neq Q(A)$.

THEOREM 5. Let $S=A C$ be the central closure of the semiprime P.I. ring $A$. Then $C=$ center $S$ hence $S$ is a $\pi$-regular ring integral over $C$. Furthermore $S=Q(A)$ if and only if $S$ is a finitely generated C-module.

Proof. Since $A \subseteq S \subseteq Q(A)$ we have $Q(S)=Q(A)$ and so $C=$ center $S$, since $C$ is the maximal quotient ring of the center of $S$. From Theorem $4, S$ is $\pi$-regular and integral over $C$ and is a finitely generated $C$-module if $S=Q(A)$ [2, Theorem 3.7]. On the other hand if $S$ is finitely generated as a $C$-module then because $S$ is a nonsingular $C$-module and $C$ is a regular self-injective ring, $S$ is then an injective 
$C$-module [11, Theorem 2.5]. However $S$ is an essential $C$-submodule of $Q(A)$ and so $S=Q(A)$.

It seems reasonable to conjecture that if $A$ is a regular ring with a polynomial identity and $M$ is a finitely generated $A$-module then $\operatorname{End}_{A}(M)$ is a $\pi$-regular ring. This is certainly true if $A$ is a commutative or if $A$ is finitely generated over its center. For $\operatorname{End}_{A}(M)$ is an integral extension of a regular subring of its center and so has its prime ideals maximal. Our concluding results are related to this conjecture.

THEOREM 6. Let $A$ be a semiprime ring with a polynomial identity and let $M$ be finitely generated flat left $A$-module. Then every $A$ endomorphism of $M$ which is onto is also one-to-one.

Proof. Let $Q$ denote the maximal quotient ring of $A$ and let $M^{*}=Q \otimes_{A} M$. Since $M$ is a flat left $A$-module the mapping from $M$ to $M^{*}$ given by $m \rightarrow 1 \otimes m$ is an $A$-monomorphism. Since $M$ is a finitely generated $A$-module, $M^{*}$ is a finitely generated $Q$-module. However $Q$ is a regular ring which is a finitely generated algebra over its center [2]. Thus $E=\operatorname{End}_{Q}\left(M^{*}\right)$ is a $\pi$-regular ring. If $f \in \operatorname{End}_{A}(M)$ then $f^{*}=1 \otimes f \in E$ and the mapping $f \rightarrow f^{*}$ is a ring isomorphism from $\operatorname{End}_{A}(M)$ into $E$, since $m \rightarrow 1 \otimes m$ is an isomorphism. Finally if $f$ is onto then $f^{*}$ is also onto. Because $E$ is $\pi$-regular, $f^{*}$ is invertible in $E$. It follows then that $f$ is one-to-one.

COROLlary 7. Let $A$ be a regular ring with a polynomial identity and $M$ a finitely generated $A$-module. Then every onto endomorphism of $M$ is one-to-one.

We remark that J. Fisher and R. Snider have constructed an example (unpublished) of a ring with a polynomial identity having a finitely generated module for which not all onto endomorphisms are one-to-one.

\section{REFERENCES}

1. E. P. Armendariz and J. W. Fisher, Regular P.I.-rings, Proc. Amer. Math. Soc., 39 (1973), $247-251$.

2. E. P. Armendariz and S. A. Steinberg, Regular self-injective rings with a polynomial identity, Trans. Amer. Math. Soc., 190 (1974), 417-425.

3. H. Bass, Finitistic dimension and a homological generalization of semiprimary rings, Trans. Amer. Math. Soc., 95 (1960), 466-488.

4. F. DeMeyer and E. C. Ingraham, Separable algebras over commutative rings, Lecture Notes in Mathematics, Vol. 181, Springer-Verlag, New York and Berlin, 1971.

5. J. W. Fisher and R. L. Snider, On the von Neumann regularity of rings with regular prime factor rings, Pacific J. Math., 54 (1974), 135-144. 
6. R. Hamsher, Commutative rings over which every module has a maximal submodule, Proc. Amer. Math. Soc., 18 (1967), 1133-1137.

7. A. G. Heinecke, A remark about noncommutative integral extensions, Canad. Math. Bull., 13 (1970), 359-361.

8. W. S. Martindale, III, Prime rings satisfying a generalized polynomial identity, J. Algebra, 12 (1969), 576-584.

9. —_, On semiprime P.I.-rings, Proc. Amer. Math. Soc., 40 (1973), 364-369.

10. G. Michler and $O$. Villamayor, On rings whose simple modules are injective, J. Algebra, 25 (1973), 185-201.

11. F. L. Sandomierski, Nonsingular rings, Proc. Amer. Math. Soc., 19 (1968), 225-230.

12. J. A. Wehlen, Algebras over absolutely flat commutative rings, Trans. Amer. Math. Soc., 196 (1974), 149-160.

Received October 14, 1975, and in revised form April 1, 1976.

University of TeXas - Austin 



\section{Pacific Journal of Mathematics}

\section{Vol. 66, No. $1 \quad$ November, 1976}

Helen Elizabeth. Adams, Factorization-prime ideals in integral domains ............ Patrick Robert Ahern and Robert Bruce Schneider, The boundary behavior of Henkin's kernel.

Daniel D. Anderson, Jacob R. Matijevic and Warren Douglas Nichols, The Krull

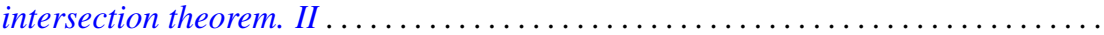

Efraim Pacillas Armendariz, On semiprime P.I.-algebras over commutative regular

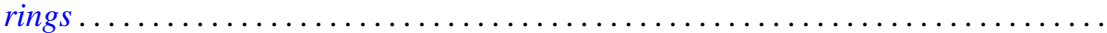

Robert H. Bird and Charles John Parry, Integral bases for bicyclic biquadratic fields

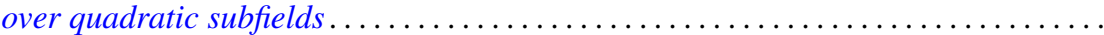

Tae Ho Choe and Young Hee Hong, Extensions of completely regular ordered

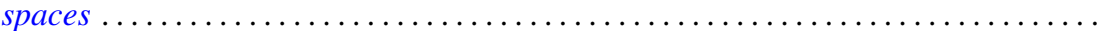

John Dauns, Generalized monoform and quasi injective modules ...............

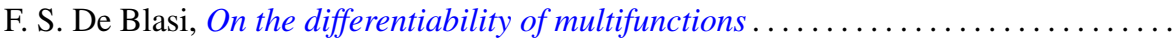

Paul M. Eakin, Jr. and Avinash Madhav Sathaye, R-endomorphisms of $R[[X]]$ are

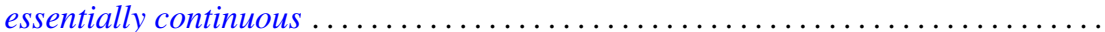

Larry Quin Eifler, Open mapping theorems for probability measures on metric

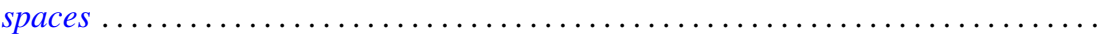

Garret J. Etgen and James Pawlowski, Oscillation criteria for second order self adjoint

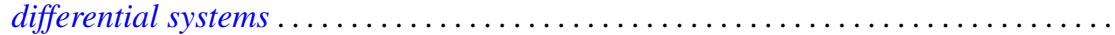

Ronald Fintushel, Local $S^{1}$ actions on 3-manifolds .

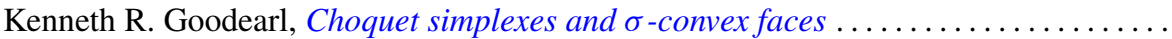

John R. Graef, Some nonoscillation criteria for higher order nonlinear differential

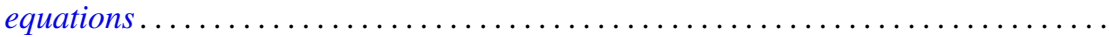

Charles Henry Heiberg, Norms of powers of absolutely convergent Fourier series: an example.

Les Andrew Karlovitz, Existence of fixed points of nonexpansive mappings in a space

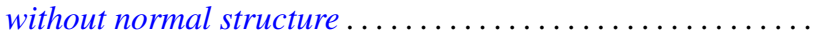

Gangaram S. Ladde, Systems of functional differential inequalities and functional

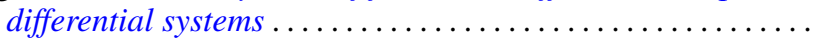

Joseph Michael Lambert, Conditions for simultaneous approximation and interpolation with norm preservation in $C[a, b]$.

Ernest Paul Lane, Insertion of a continuous function.

Robert F. Lax, Weierstrass points of products of Riemann surfaces .

Dan McCord, An estimate of the Nielsen number and an example concerning the Lefschetz fixed point theorem...

Paul Milnes and John Sydney Pym, Counterexample in the theory of continuous functions on topological groups...

Peter Johanna I. M. De Paepe, Homomorphism spaces of algebras of holomorphic functions

Judith Ann Palagallo, A representation of additive functionals on $L^{p}$-spaces,

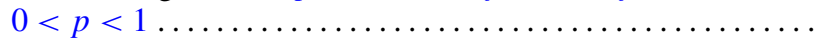

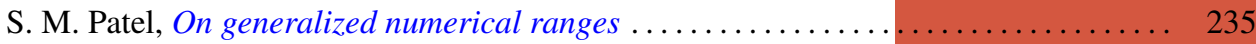

Thomas Thornton Read, A limit-point criterion for expressions with oscillatory

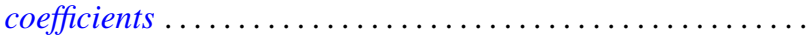

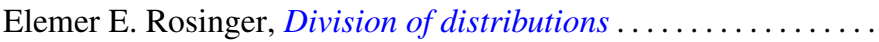

Peter S. Shoenfeld, Highly proximal and generalized almost finite

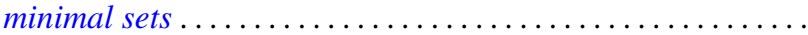

R. Sirois-Dumais and Stephen Willard, Quotient-universal sequential spaces

Robert Charles Thompson, Convex and concave functions of singular values of matrix sums....

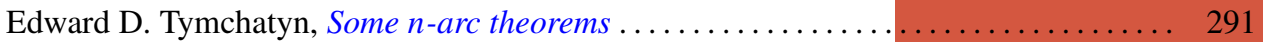

